Forum - Kommentar

\title{
Zu den zehn Argumenten für dialogische Interaktion und kognitive Modelle von Helmut Hildebrandt
}

\author{
Kommentar aus der neuropsychologischen Praxis \\ in Klinik und Ambulanz
}

Volker Völzke

Leitung Therapie, Psychologie, Neuropsychologie, VAMED Klinik Hattingen

\section{Einleitung}

Der umfassende, innovative und sehr erfreuliche Artikel von Helmut Hildebrandt (2021) wird ähnlich dem Artikel von Gauggel (2003) Diskussionen auslösen und hoffentlich weitere klinische Forschung initiieren bzw. Fortbildungen bereichern. Hildebrandt (2021) wirft Fragen auf, die häufig in der Alltagspraxis mit vielen Routinen bzw. Grenzen untergehen, zumal die aktuelle neuropsychologische Forschung oft gekennzeichnet ist durch die Fokussierung auf eher akademische Fragestellungen.

Aktuell werden aber durch die Weiterbildungsordnung und den neuen Studiengang Psychotherapie die Weichen für die nächste Generation klinischer Neuropsychologinnen und -psychologen der Praxis gestellt: Neuropsychologische Psychotherapie steht zukünftig neben den etablierten psychotherapeutischen Verfahren.

Die jeweiligen Überschriften der Argumente initiieren eine spontane, auch teilweise emotionale Reaktion und Kommentare. An diesen möchte ich Sie teilhaben lassen.

\section{Diagnostik}

Neuropsychologische Diagnostik simuliert gestuft die Alltagswelt, so vereinfacht Hildebrandt. Sie ermöglicht eine "geführte" Selbsterfahrung und -überprüfung der Betroffenen. Tests reduzieren Alltagskomplexität auf basale Grundmechanismen. Wichtig ist es aus meiner Sicht, die- sen Aspekt den Betroffenen und ihren Angehörigen gegenüber immer wieder transparent zu machen („Es ist wichtig, dass Sie wissen, was Sie weiterhin können und was durch die Verletzung problematisch geworden ist.").

\section{Unawareness}

Die Überschrift mit dem Hinweis auf ein „Recht auf Unawareness" hat mich beim ersten Lesen besonders irritiert und spontan Widerspruch ausgelöst. Denn die fehlende Krankheitseinsicht belastet die Rehabilitation und bei einem chronischen Verlauf insbesondere die Angehörigen in besonderer Weise (Gauggel, 2016). Bei intensiverer Lektüre offenbart sich aber die Sinnhaftigkeit der Aussage. Wichtig ist ein „wertschätzendes geleitetes Entdecken“ oder - in der Terminologie von Hildebrandt (2021) - die „Cues“. Diese Haltung spricht aus meiner Sicht gegen eine starre Standarddiagnostik ohne individuelle Variation. Diagnostik ist Therapie, indem sie auch Awareness im Sinne von Selbstentdecken erst ermöglicht. Ergänzen möchte ich den aus meiner Sicht notwendigen Aspekt der Selbsteffizienz, welcher insbesondere in psychotherapeutischen Ansätzen Bedeutung besitzt (u.a. Sheeran et al., 2016). Betroffene aber auch Angehörige bedürfen eines Wissens um hilfreiche bzw. sinnhafte Therapien, individuelle Grenzen, Rahmenbedingungen und hilfreiche Kognitionen („Was hilft mir und was tut mir gut?"). Dazu gehört ergänzend auch wesentlich der Aspekt der Selbstfürsorge (Pausenmanagement etc.). 


\section{Wissenschaftliche Fundierung}

Hildebrandt (2021) fordert eine neurowissenschaftliche Fundierung der Therapie. Er erläutert das sehr plastisch am Beispiel der Wortfindungsstörungen bei Aphasie. Eine so detaillierte Analyse ist für diverse Störungsbilder oder Symptomkomplexe sehr hilfreich. Besonders gut gefallen hat mir der Hinweis der "geschützten Umgebung" in der Therapie. Scham, Kränkung und Entmutigung sind zu vermeiden. Parallel geht es um eine Gradwanderung im Rahmen der Konfrontation mit dem Defizit. Im therapeutischen Kontakt bedarf es "hilfreicher" Formulierungen („Es ist ganz typisch, dass Betroffene mit dieser Art der Schädigung in diesem Bereich Probleme haben. Bewährt haben sich ...").

Natürlich ist, so Hildebrandt, der Schweregrad der Störung bzw. die Phase in den Interventionen bedeutsam. Hoch praxisrelevant ist der weitere Hinweis, dass reine Bottom-up-Therapien bisher keine wissenschaftliche Evidenz aufweisen. Sie sind, so bewertet er aus meiner Sicht korrekt, aber eine hilfreiche Unterstützung für die neuropsychologische Therapie. Medikation, tDCS (transkranielle Gleichstromstimulation) oder ergänzend TMS (transkranielle Magnetstromstimulation) und optokinetische Stimulation sind hier zu erwähnen und einer weiteren wissenschaftlichen Evaluation und auch Praxisevaluation zu unterziehen.

Kognitive Modelle bzw. psychologische Theorien ermöglichen die Weiterentwicklung und Modifikation der neuropsychologischen Therapie. Am Beispiel der Therapie von Gedächtnisdefiziten und bei der Neglecttherapie gelingt das Hildebrandt (2021) modellhaft sehr hilfreich. Beispielhaft wird das interaktive "Cueing" in der Therapie fokussiert (i.S. der Therapie an der Leistungsgrenze). Entsprechende Konzepte zu den wichtigsten Störungsbildern werden im klinischen Alltag oft übersehen oder ignoriert. Hilfreich ist auch die Bewertung des Einsatzes von „Virtual Reality" (VR). VR an sich bietet keinen Vorteil, ist sogar eher „blind“, wenn nicht der individuell sinnvolle Kontext ausgewählt wird. Eine zu komplexe Umgebung, wie sie oft im Spielkontext gewählt wird, ist insbesondere in der Phase der frühen Rehabilitation möglicherweise verwirrend oder sogar „schädlich“.

An dieser Stelle fehlt mir der Hinweis auf die Bedeutung der therapeutischen Beziehung bzw. Allianz als bedeutsamer Wirkfaktor (Völzke, 2021). „Cues“ in der virtuellen oder physikalischen Realität sind nur ein essenzieller Aspekt der neuropsychologischen Therapie. Am Beispiel des „Fading out" bei der Therapie von Gedächtnisstörungen und Neglect erläutert Hildebrandt (2021) wiederum plakativ die sinnvollen Stufen bis zur Selbstständigkeit der Betroffenen. Wichtig ist hierbei die Ergänzung, dass existierende oder sich entwickelnde „basale“ Arbeitsgedächtniskompetenzen für die erfolgreiche Therapie bis hin zur Selbstständigkeit fundamental sind.

\section{Prognosestellung}

Zentral für Hildebrandt (2021) ist die wissenschaftlich fundierte Prognosestellung unter Berücksichtigung des Zeitraums nach dem Ereignis, der Größe und Lage der Läsion, des Alters, der erreichten Einsicht und der psychosozialen Ressourcen. An dieser Stelle wäre ein Hinweis auf das Modell der Internationalen Klassifikation der Funktionsfähigkeit, Behinderung und Gesundheit (ICF; Fries, Reuther \& Lössl, 2017) und die dann später im Artikel folgende Berücksichtigung des Läsionsmechanismus (bspw. negative Prognose bei Hypoxie) hilfreich. Pragmatisch ist der Hinweis auf eine zyklische Planung der Therapiesitzung mit dem herausfordernden Mittelteil. In der Ausund Weiterbildung ist es sinnvoll, u.a. den Hinweis zu geben, dass die Sitzung möglichst mit einem Erfolg beendet werden soll (u.a. Recency-Effekt).

Hildebrandt (2021) weist daraufhin, dass die Kombination von Intervention bei komplexen Störungsbildern bisher völlig unklar ist bzw. ein zukünftiges Forschungsfeld darstellt. Relativ spät im Artikel diskutiert werden die Bedeutung der vulnerablen Phasen (akut vs. chronisch) im rehabilitativen Kontext. Während postakut die funktionellen Folgen (Funktionsdefizite) im Vordergrund stehen, so ist es später die Krankheitsbewältigung (neuropsychologische Psychotherapie). Sehr alltagsrelevant ist der Hinweis auf Überforderung im akuten Stadium und bei sehr großen Läsionen (siehe Mediainfarkten). Es folgt nochmals der Hinweis auf die Effektivität einer Add-onTherapie (s.o.) bei einem spezifischen kognitiven Modell. Ergänzen möchte ich an dieser Stelle den sehr praxisrelevanten Hinweis auf die sog. Reservekapazität (Perneczky et al., 2019), der nicht nur in der Demenzforschung Relevanz besitzt.

Im klinischen Alltag spielen nach meiner Erfahrung insbesondere Komorbiditäten und assoziierte psychische Störungen eine extrem bedeutsame Rolle (prämorbide Depression etc.). Sie bestimmen oft den Verlauf der Therapie bzw. Rehabilitation. Herausragend wichtig ist der Hinweis von Hildebrandt (2021) auf die Notwendigkeit der Änderung des Fokus nach intensiver ca. 3-wöchiger Therapie, falls keine Verbesserungen eintreten. Dieser und andere erwähnte Aspekte sollten integraler Bestandteil von wissenschaftlich fundierter Fortbildung für alle therapeutischen Gruppen und insbesondere Ärztinnen und Ärzte sein.

\section{Wünsche und Perspektiven}

Wünschenswert wäre eine Visualisierung der Argumente mit einer Abbildung der Einflussfaktoren. Für die klinische Praxis (insbesondere für Berufsanfängerinnen und 
-anfänger) erscheint eine Checkliste hilfreich, welche die oben genannten Aspekte transparent macht und eine Priorisierung ermöglicht. Aus meiner Sicht bietet das ICFModell (Fries et al., 2017) parallel zum sehr gelungenen Praxisbuch von Winson, Wilson und Bateman (2020) mit den Argumenten von Hildebrandt (2021) einen hilfreichen Rahmen für neuropsychologische Therapie.

Weiterhin ist die klinische Praxis der Neuropsychologie, wie auch erwähnt, sehr phasenspezifisch. Akutklinik, Frührehabilitation, Anschlussrehabilitation, Tagesklinik und Praxis/Ambulanz erfordern sehr spezifische Herangehensweisen. Insgesamt bietet der Artikel einen außerordentlich wertvollen Beitrag für die Weiterentwicklung der „klinischen" Neuropsychologie.

\section{Literatur}

Fries, W., Reuther, P. \& Lössl, H. (Hrsg.). (2017). Teilhaben!! Neurorehabilitation und Nachsorge zu Teilhabe und Inklusion. Bad Honnef: Hippocampus.

Gauggel, S. (2003). Grundlagen und Empirie der Neuropsychologischen Therapie: Neuropsychotherapie oder Hirnjogging? Zeitschrift für Neuropsychologie, 14, 217-246.

Gauggel, S. (2016). Störungen der Krankheitseinsicht (Fortschritte der Neuropsychologie, Bd. 17). Göttingen: Hogrefe.

Hildebrandt, H. (2021). Zehn Argumente für dialogische Interaktion und kognitive Modelle als Grundlage neuropsychologischer Rehabilitation. Zeitschrift für Neuropsychologie, 32 (4), 229-242.
Perneczky, R., Kempermann, G., Korczyn, A., Matthews, F., Arfan Ikram, M., Scarmeas, N. et al. (2019). Translational research on reserve against neurodegenerative disease: Consensus report of the International Conference on Cognitive Reserve in the Dementias and the Alzheimer's Association Reserve, Resilience and Protective Factors Professional Interest Area working groups. BMC Medicine, 17, 47.

Sheeran, P., Maki, A., Montanaro, E., Avishai-Yitshak, A., Bryan, A., Klein, W.M.P. et al. (2016). The impact of changing attitudes, norms, and self-efficacy on health-related intentions and behavior: A meta-analysis. Health Psychology, 35, 1178-1188.

Völzke, V. (2021). Therapeutische Beziehung in der Neuropsychologie. Beitrag in Vorbereitung zur Publikation. PiD - Psychotherapie im Dialog.

Winson, R., Wilson, B.A. \& Bateman, A. (2020). Rehabilitation nach Hirnschädigung. Ein Therapiemanual. Göttingen: Hogrefe.

\section{Historie}

Eingereicht: 15. September 2021

Akzeptiert: 15. September 2021

\section{Interessenkonflikt}

Der Autor hat keine Interessenkonflikte.

\section{Dr. rer. medic. Volker Völzke}

VAMED Klinik Hattingen

Am Hagen 20

45527 Hattingen

Deutschland

volker.voelzke@vamed-gesundheit.de 